\title{
Multi-Client Verifiable Computation with Stronger Security Guarantees
}

\author{
S. Dov Gordon ${ }^{1}$, Jonathan Katz ${ }^{2}$, Feng-Hao Liu ${ }^{2}$, \\ Elaine $\mathrm{Shi}^{2}$, and Hong-Sheng Zhou ${ }^{3}$ \\ 1 Applied Communication Sciences, USA \\ sgordon@appcomsci.com \\ 2 University of Maryland, USA \\ \{jkatz, fenghao, elaine\}@cs. umd. edu \\ 3 Virginia Commonwealth University, USA \\ hszhou@vcu.edu
}

\begin{abstract}
At TCC 2013, Choi et al. introduced the notion of multiclient verifiable computation (MVC) in which a set of clients outsource to an untrusted server the computation of a function $f$ over their collective inputs in a sequence of time periods. In that work, the authors defined and realized multi-client verifiable computation satisfying soundness against a malicious server and privacy against the semi-honest corruption of a single client. Very recently, Goldwasser et al. (Eurocrypt 2014) provided an alternative solution relying on multi-input functional encryption.

Here we conduct a systematic study of MVC, with the goal of satisfying stronger security requirements. We begin by introducing a simulationbased notion of security that provides a unified way of defining soundness and privacy, and automatically captures several attacks not addressed in previous work. We then explore the feasibility of achieving this notion of security. Assuming no collusion between the server and the clients, we demonstrate a protocol for multi-client verifiable computation that achieves stronger security than the protocol of Choi et al. in several respects. When server-client collusion is possible, we show (somewhat surprisingly) that simulation-based security cannot be achieved, even assuming only semi-honest behavior.
\end{abstract}

\section{Introduction}

Protocols for verifiable computation (or secure outsourcing) allow computationally weak clients to delegate to a more powerful server the computation of a function $f$ on a series of dynamically chosen inputs $x^{(1)}, x^{(2)}, \ldots$. The main desideratum is that, following a pre-processing stage whose complexity depends on $f$, the work of the client per function evaluation should be significantly lower than the cost of computing the function itself [20]. The initial proposal and construction of non-interactive verifiable computation [20] led to a long line of follow-up work [1, 3, 7-10, 16 18, 21, 26, 27, 33 36].

Y. Dodis and J.B. Nielsen (Eds.): TCC 2015, Part II, LNCS 9015, pp. 144-168, 2015.

(C) International Association for Cryptologic Research 2015 
We are interested here in the multi-client setting introduced by Choi et al. [15]. Imagine that $n$ clients wish to compute some function $f$ over their joint inputs $\left\{\left(x_{1}^{\text {(ssid) }}, \ldots, x_{n}^{\text {(ssid) }}\right)\right\}_{\text {ssid }}$ for a series of subsessions identified by ssid. (One can view the ssid as encoding a current time period, though there are other possibilities as well.) As in earlier work, we assume no client-client communication, and focus on non-interactive solutions in which each evaluation of the function requires only a single round of communication between each client and the server.

In earlier works on multi-client verifiable computation [15, 24], the primary goal is to achieve security (soundness and privacy) against a malicious server, assuming that clients behave honestly. Soundness means that a malicious server should not be able to fool a client into accepting a wrong result; privacy means that clients' inputs should remain hidden from the server. (Choi et al. also considered privacy against clients, but while still assuming semi-honest client behavior.)

\subsection{Our Contributions}

In this paper, we conduct a systematic study of multi-client verifiable computation with stronger security guarantees. The primary question we address is security when clients may be malicious. These malicious clients may potentially be colluding with each other, or with the server.

Formal Security Modeling. We begin by introducing a simulation-based notion of security in the universal composability framework, which provides a unified way of defining soundness and privacy. As a technical advantage, it means that protocols satisfying the definition achieve a strong, simulation-based notion of security not considered in previous work. Our definition also automatically captures adaptive soundness as well as selective-failure attacks, which were not handled in prior work on the multi-client setting 1 .

Impossibility When the Server and Clients Collude. Ideally, one would like to achieve a strong notion of security where a subset of the clients may be

\footnotetext{
${ }^{1}$ Intuitively, a scheme suffers from selective-failure attacks if the server can learn some secret information from the "decision" of the clients, upon receiving output from the server. In the single-client setting, previous schemes in the work [16, 20] can be completely broken by the attacks, unless the clients are willing to redo the expensive preprocessing upon any server failure. In the multi-client setting, the same attacks also apply to the scheme by Choi et al. [15], which is basically an extension of [20]. We note that there is no simple fix to the approaches taken in [15, 16, 20] using known techniques. Previous schemes that are not vulnerable to such attacks (such as the scheme in [36]) used completely different approaches.

Adaptive soundness is a technical issue pointed out by Bellare et al. [5] - if a Yao's garbled circuit is published first and later the adversary can choose inputs based on the garbled circuit, then it is not known how to prove security other than just assuming the garbling scheme itself is secure. Previously, the schemes of [15, 20] used Yao's garbled circuits in this way, so the schemes suffer from such drawback. See the work of Choi et al. [15] for further discussions about adaptive soundness and selective failure attacks.
} 
corrupted, and may be colluding with the server. Unfortunately, we show that simulation-secure MVC is impossible to realize (for general functions) when the server colludes with clients. This impossibility result holds even in the standalone setting, even when the server colludes with only a single, semi-honest client, and even in the presence of trusted setup assumptions such as PKI, common reference strings (CRS), shared secret randomness, etc. Intuitively, our lower bound result is due to a connection we establish between MVC and virtual black-box (VBB) obfuscation, whose impossibility is known [4]. More details can be found in Section 5 .

Feasibility Result: When Server and Clients do not Collude. In contrast to the above, we show positive results for the case when client-server collusion is assumed not to occur. We show a construction that achieves security (i.e., soundness and privacy) against either a malicious server, or an arbitrary set of malicious, colluding clients. Our construction achieves both adaptive soundness and security against selective abort.

Our construction relies only on falsifiable assumptions. While it is alternatively possible to construct MVC schemes using a new notion, multi-input functional encryption, by Goldwasser et al. [24], this notion inherently requires (indistinguishable) obfuscation, which requires non-falsifiable assumptions or exponential assumptions [22]. Moreover, current constructions of obfuscation have prohibitively large overhead.

\subsection{Techniques and New Primitives}

Techniques used for achieving our upper bound results can be of independent interest. When server-client collusion is not allowed, we take a two-step approach to achieve simulation-security of MVC. As a stepping stone, we identify a new building block named multi-sender attribute-based encryption (mABE).

Our Two-Step Approach for MVC. We start with a protocol which achieves the simulation-based security against either (i) a malicious server or (ii) any coalition of semi-honest clients. Although this is also achieved by the protocol of Choi et al. 15] - even if not claimed explicitly there - our construction has the advantages of offering adaptive soundness based on standard assumptions as well as resilience to selective-failure attacks.

We then present a generic compiler that upgrades our intermediate solution (as well as the one by Choi et al. [15]) to handle an arbitrary subset of malicious clients. While we could rely on standard techniques, distributing commitments to random tapes during setup, and asking each party to prove in zero knowledge that they have acted honestly, we instead offer a compiler that does not require committed randomness, allowing us to reduce our setup assumptions to a simple common reference string. We demonstrate that as long as our semi-honest protocol offers a sufficiently strong notion of privacy, our compiler provides security against malicious corruption. This gives us a non-interactive multi-client verifiable computation protocol secure against a malicious adversary under all possible cases of non-client-server collusions, in the standard model under falsifiable assumptions. 
A New Building Block: mABE. We identify a new building block, multi-sender attribute-based encryption, which can be of independent interest.

Recall that in the single sender setting, Parno et al. 36] showed that an attribute-based encryption (ABE) (that supports functions and their complements) implies publicly verifiable computation (without input privacy). Later, Goldwasser et al. [26] showed (i) how to compile an ABE scheme to a privateindex functional encryption scheme using fully homomorphic encryption (FHE), and (ii) that private-index functional encryption implies input-private publicly verifiable computation.

We conduct a parallel study in the multi-sender setting. The multi-sender counterpart, multi-sender ABE (mABE) is defined as follows. Each sender $\mathrm{P}_{i} \in$ $\left\{\mathrm{P}_{1}, \ldots, \mathrm{P}_{n}\right\}$ has an attribute value $x_{i}$, as well as two input messages $\left(m_{0}^{(i)}, m_{1}^{(i)}\right)$. A single receiver (or server) can use a decryption key for function $f_{i}$ to learn $m_{b}^{(i)}$ if and only if $b=f_{i}\left(x_{1}, \ldots, x_{n}\right)$. We show how to construct an mABE scheme secure against a malicious server or semi-honest senders. To construct this mABE scheme, we first observe a special "local encoding" property of the LWE-based ABE scheme by Gorbunov, Vaikuntanathan, and Wee 30]. We then combine this observation with a proxy-OT protocol proposed by Choi et al. [15].

After obtaining the mABE construction, we then apply Goldwasser et al's compiler techniques [26] to transform it into an attribute-hiding mABE scheme (which can also be thought of as a multi-sender, private-index functional encryption scheme). Finally, just as single-sender private-index functional encryption implies input-private verifiable computation, we show that attribute-hiding mABE implies multi-client verifiable computation with input privacy, secure against a malicious server or an arbitrary subset of semi-honest clients. We can then use the compiler described previously to obtain security in the face of malicious corruptions, so long as there is no client-server collusion.

Sacrificing Input Privacy to Allow Server-Client Collusion. Since attribute hiding mABE implies multi-client verifiable computation, it follows that attribute hiding $m A B E$ is also impossible for general functions under server-client collusion. However, it is still interesting to consider settings without input privacy/attribute hiding. In this work, we show that any mABE (without attribute hiding) construction that is secure under some arbitrary corruption pattern implies public input MVC under the same corruption pattern; in particular, this gives a method of handling server-client collusion. We also show that an mABE scheme secure under server-client collusion, even in the standalone setting, implies extractable witness encryption (equivalently, point-filter obfuscation) 25]. So building MVC protocols without input privacy via this method would inherently require non-falsifiable assumptions. We note that it is also possible to construct MVC protocols without input privacy against an arbitrary corruption based on other non-falsifiable assumptions, such as SNARKs. It is an interesting question - whether we can construct a secure MVC without input privacy against an arbitrary corruption based on falsifiable assumptions, yet one should keep in mind that any possible solution should avoid the route using mABE, as implied by the result above. 


\subsection{Related Work}

Non-interactive verifiable computation was first proposed by Gennaro, Gentry, and Parno 20]. Since then, various improvements have been proposed [1, 16, 17, 21, 27, 36], and constructions for specific functionalities [9, 18, 34, 35].

Various works have considered server-aided secure computation with the goal of eliminating client-to-client interaction. Most of these existing works do not achieve complete non-interactivity, in the sense that they still require multiple rounds of server-client interaction. Kamara et al. [31, 32] consider server-aided multi-party computation, but their approach is not non-interactive.

Multi-input functional encryption [24] is also related to non-interactive multiparty outsourcing. The earlier work by Shi et al. [37] shares similar goals, but for specific functionalities such as summation and variance. Shi et al. 37] also describe various application domains such as secure sensor network aggregation. In the multi-input functional encryption model or that of Shi et al. [37], the server learns the final outcome of the computation, and verifiability (i.e., soundness) is not an inherent part of the problem formulation. Interestingly, Goldwasser et al. 24], observe that multi-input functional encryption can, in fact, be leveraged to construct multi-client verifiable computation. This solution uses the technique developed in [36] which solves the selective-failure issue. However, Goldwasser et al. [24] do not consider malicious clients or client-server collusion. In addition, another major drawback is that known multi-input functional encryption schemes rely on nonfalsifiable assumptions related to obfuscation, and this is somewhat necessary as pointed out by [24].

\section{Multi-Client Verifiable Computation}

\subsection{Definitions}

We start by introducing the notion of non-interactive multi-client verifiable computation (MVC) that has the following structure: let $\kappa$ be the security parameter, $n$ be the number of clients $\mathrm{P}_{1}, \ldots, \mathrm{P}_{n}$ who are delegating some computation on some $n$-ary function $f: \mathcal{X}^{n} \rightarrow \mathcal{Y}^{n}$ to a distinguished server Serv and would like to verify the correctness of their answers. Here we assume each client's input message space is $\mathcal{X}$, and output message space $\mathcal{Y}$, for some polynomial-length (in the security parameter) $|\mathcal{X}|$ and $|\mathcal{Y}|$.

Intuitively, MVC protocols have the following properties: (1) All participants are allowed to access to a certain initial setup $\mathcal{G}$ (e.g., PKI, CRS). (2) Then an offline stage follows; in the offline stage, each client sends a single message to the server Serv. (3) In the online stage, in a single time period (subsession), each client is only allowed to send an outgoing message to the server and then receive an incoming message from the server. In the whole paper, we assume that the clients cannot communicate with each other directly, and can only send a single round of message to the server per time period (subsession). Next, we give more details. 
Definition 1 (Non-interactive Multi-client Verifiable Computation). Let $\kappa$ be the security parameter, $n$ be the number of clients and $f$ be an $n$-ary function being computed. A non-interactive multi-client verifiable computation consists of $n$ clients $\mathrm{P}_{1} \ldots \mathrm{P}_{n}$ and a server Serv with the following structure:

Setup stage: All parties $\mathrm{P}_{i}$ 's, $i \in[n]$ and Serv have access to a setup $\mathcal{G}$, where party $\mathrm{P}_{i}$ obtains (pub, $\mathrm{sk}_{i}$ ) upon queries for some secret and public information.

Offline stage: Each client $\mathrm{P}_{i}$ sends a single message to the server. The server stores these as $\hat{f}$, an encoded version of $f$.

Online stage: This step is a query-response move: at each sub-session (or time period) ssid, upon receiving an input (ssid, $\left.x_{i}\right)$ for $i \in[n]$, the client $\mathrm{P}_{i}\left(\mathrm{pub}, \mathrm{sk}_{i}, x_{i}\right)$ computes some message $\left(\hat{x}_{i}, \tau_{i}\right)$. Then he sends $\hat{x}_{i}$ to the server and stores $\tau_{i}$ as a secret.

The server Serv carries out the computation on the messages received, and sends each client $\mathrm{P}_{i}$ for $i \in[n]$ an encoded output (ssid, $\hat{y}_{i}$ ).

Each client computes and some output $y_{i} \cup\{\perp\}$ based on (pub, sk $\left.\mathrm{k}_{i}, \hat{y}_{i}, \tau_{i}\right)$, where $\perp$ means that he is not convinced with the outcome.

Remark 1. For the setup $\mathcal{G}$, we do not specify whether it is trusted in our definition. For our positive results, we want to minimize the requirements, and we showed that a self-registered PKI is enough for semi-honest client or malicious server corruptions. For the case of malicious clients corruptions, we further need an additional CRS. On the other hand, for our lower bound results, we rule out a large class of instantiations of $\mathcal{G}$, including the trusted PKI, CRS, shared secret randomness, and their combinations.

Note that the trusted PKI is a setup where a trusted party generates public- and secret-key pairs for each user, and publishes the public keys to all users. The selfregistered PKI is a weaker setup where each user generates their own key pairs, and registers the public keys with the setup so that the setup can publish the public keys to all users.

\subsection{Security Definition}

The security definition for non-interactive multi-client verifiable computation, MVC, turns out to be subtle. An MVC protocol cannot achieve the standard multi-party computation security, which requires that malicious clients have only one chance to provide their inputs, and cannot switch inputs later. In the non-interactive setting, if the server and some clients are simultaneously corrupted, then after gathering the transcripts of the honest clients, by definition the malicious clients can now select different inputs for themselves and learn the corresponding outputs. For example, consider $n=2$. If client $\mathrm{P}_{1}$ and the server are corrupted, then they effectively have access to oracle $f_{1}\left(*, x_{2}\right)$ where $f_{1}$ is the output of the first party, and $x_{2}$ is the honest input of $\mathrm{P}_{2}$. The notation $*$ means that client $\mathrm{P}_{1}$ can choose arbitrary inputs for itself and query this oracle a polynomial number of times. So our security definition would allow the adversary to learn $f_{1}\left(*, x_{2}\right)$ in the ideal world, and guarantees that this is the most that he can learn. 


\section{Multi-Client Private Verifiable Computation}

The functionality is parameterized with an $n$-ary function $f: \mathcal{X}^{n} \rightarrow$ $\mathcal{Y}^{n}$. The functionality interacts with $n$ clients $\mathrm{P}_{i}$ for $i \in[n]$, a distinguished server Serv, and the simulator Sim.

\section{Initialization:}

Upon receiving (Init) from client $\mathrm{P}_{i}$, send (Init, $\mathrm{P}_{i}$ ) to notify the simulator $\mathcal{S}$ im. Later, when $\mathcal{S}$ im returns $\left(\right.$ Init, $\left.\mathrm{P}_{i}\right)$, send a notification (Init, $\mathrm{P}_{i}$ ) to the server Serv.

Upon receiving (Init) from the server Serv, send (Init, Serv) to notify the simulator $\mathcal{S}$ im.

\section{Computation:}

Upon receiving (Input, ssid, $x_{i}$ ) from client $\mathrm{P}_{i}$, send (ssid, $\mathrm{P}_{i}$ ) to notify $\mathcal{S} i m$. Later, when $\mathcal{S} i m$ returns (ssid, $\mathrm{P}_{i}$ ), store (ssid, $x_{i}$ ), and send a notification (Input, ssid, $\mathrm{P}_{i}$ ) to server Serv.

Upon receiving (Input, ssid, 1) from server Serv, retrieve (ssid, $x_{i}$ ) for all $i \in[n]$. If some (ssid, $x_{i}$ ) has not been stored yet, send (Output, ssid, fail) to the server and all clients.

- Server is not corrupted: Compute $\left(y_{1}, \ldots, y_{n}\right) \leftarrow$ $f\left(x_{1}, \ldots, x_{n}\right)$. Later when $\mathcal{S} i m$ returns (ssid, $\left.\mathrm{P}_{i}, \phi\right)$, if $\phi=$ ok, send (Output, ssid, $y_{i}$ ) to client $\mathrm{P}_{i}$; if $\phi=$ fail, send (Output, ssid, fail) to client $\mathrm{P}_{i}$.

- Server is corrupted: Let $\mathcal{I} \subseteq[n]$ denote the set of indices corresponding to corrupted clients. Let $\overline{\mathcal{I}}:=[n] \backslash \mathcal{I}$. Let $\mathbf{x}_{\mathcal{I}}^{*}$ denote the corrupted clients' inputs, $\mathbf{x}_{\overline{\mathcal{I}}}$ denote the remaining clients' inputs. Without loss of generality, we can renumber the clients such that $\mathcal{I}:=\{1,2, \ldots,|\mathcal{I}|\}$.

The functionality provides to $\mathcal{S}$ im blackbox oracle access to the following oracle $O_{f, \mathcal{I}}$ where $\mathcal{S} i m$ can choose inputs $\mathbf{x}_{\mathcal{I}}^{*}$ for corrupted clients to query:

Oracle $O_{f, \mathcal{I}}\left(\mathbf{x}_{\mathcal{I}}^{*}\right):$

Compute $\left(y_{1}, \ldots, y_{n}\right) \leftarrow f\left(\mathbf{x}_{\mathcal{I}}^{*}, \mathbf{x}_{\overline{\mathcal{I}}}\right)$.

Output $\left\{y_{i}\right\}_{i \in \mathcal{I}}$ to $\mathcal{S} i m$, and internally remember the last seen $\left\{y_{i}\right\}$ for $i \in \overline{\mathcal{I}}$.

At any time (not necessarily simultaneously for all $i$ ), on receiving (ssid, $\mathrm{P}_{i}, \phi$ ) from $\mathcal{S} i m$ for some $i \in \overline{\mathcal{I}}$, the functionality ${ }^{a}$ sends to $\mathrm{P}_{i}$ (Output, ssid, $y_{i}$ ) corresponding to the last seen $y_{i}$ if $\phi=$ ok, otherwise it sends (Output, ssid, fail) to $\mathrm{P}_{i}$.

${ }^{a}$ Restricting to sending the last seen outputs does not lose generality, since the simulator can always repeat a previous query to the oracle $O_{f, \mathcal{I}}$.

Fig. 1. Functionality $\mathcal{F}_{\mathrm{p} V \mathrm{C}}$ 
On the other hand if interaction is allowed, it is well-understood that this issue can be avoided by standard techniques.

Based on this observation, we formally define the ideal functionality for private MVC in Figure 1 that captures the above issues, and soundness and privacy. The security of the protocol above follows the standard real/ideal paradigm [28, 29]. Here we only include the universal composability (UC) definition by Canetti [13, 14]. The standalone security definition can be found in [12, 23].

Definition 2 (UC Security [14]). We say a protocol $\Pi$ securely realizes $\mathcal{F}$ if for any PPT adversary $\mathcal{A}$ in the real world, there exists a PPT simulator $\mathcal{S} i m$ in the ideal world, so that no PPT environment $\mathcal{Z}$ is able to tell the real world execution from the ideal world execution, i.e., $\operatorname{EXEC}_{\mathcal{A}, \Pi, \mathcal{Z}} \approx \operatorname{EXEC}_{\mathcal{S i m}, \mathcal{F}, \mathcal{Z}}$.

We can also define a notion of verifiable computation without input privacy. This is essentially the same definition, except that the server learns all the inputs of the clients. We present a formal description and provide a construction of this relaxed notion in the full version of this paper. In the following remarks we highlight and clarify a few properties of the stronger definition above:

Soundness Against Selective Failure Attacks: Our ideal-functionality models a reactive functionality that has multiple sub-sessions after a common preprocessing (i.e., Initialization) phase. Our definition implies this soundness, where learning the decision bit of the clients does not help the server to fool the clients. In particular, following the convention of simulation-based definition, our security definition requires the clients to report the outputs (and acceptance decisions) to the environment.

Communication Model. We assume that the adversary controls the communication medium between all parties. Our protocol later relies on PKI setup, and we can implement a secure channel with PKI. Therefore, while not explicitly stated, all our protocols are described assuming the secure channel ideal world.

Semi-Honest v.s. Malicious Corruption. Semi-honestly corrupted participants follow the protocol faithfully, but the adversary sees the internal states of all semi-honestly corrupted parties.

As mentioned above, due to the non-interactive nature, if the server and at least one client are simultaneously corrupted either in the malicious or semi-honest model, then our ideal functionality $\mathcal{F}_{\mathrm{p} v \mathrm{c}}$ implements a blackbox-access oracle which the simulator can query multiple times by specifying inputs for the malicious clients. For malicious corruption, the simulator can ask the ideal functionality to send outputs to different clients corresponding to different corrupted clients' inputs. For example, suppose $\mathrm{P}_{1}$ and the server are maliciously corrupted, the simulator can ask the functionality to send $f_{2}\left(x_{1}, x_{2}, x_{3}\right)$ to $\mathrm{P}_{2}$, and send $f_{3}\left(x_{1}^{\prime}, x_{2}, x_{3}\right)$ to $\mathrm{P}_{3}$. For semi-honest corruption, the outputs sent back to the clients always correspond to inputs chosen by the environment. 
Static Corruption. We assume a static corruption model in this paper, where some protocol participants are corrupted at the beginning of protocol execution.

UC and Stand-Alone Security. In the paper we use both the UC definition and standalone security definition. In the standalone security, the environment machine $\mathcal{Z}$ (i.e., the distinguisher) provides inputs to all protocol participants and the adversary at the beginning of protocol execution, and it receives outputs from these entities when the execution is complete. The environment and the adversary are not allowed to communicate during the protocol execution. Protocols secure in the standalone security model can be composed sequentially. On the other hand, in the UC framework, the environment and the adversary are always allowed to communicate. Protocols secure in the UC framework can be composted with arbitrary protocols. It is obvious that UC security implies stand-alone security.

Efficiency. An important feature of MVC is the online efficiency of the clients. Usually, we require the clients' computation time be much less than the complexity of the function $f$, so that over many online computations, the total cost of the clients will have low amortized cost. However, for private MVC, in some cases it is also interesting if the clients' computation time is similar to $f$, e.g. when the function $f$ is simple. For example, it client $\mathrm{P}_{1}$ and $\mathrm{P}_{2}$ want to do a secure comparison over their inputs. The privacy requirement makes it interesting regardless of whether the clients' online computation time is smaller than the function being delegated. We do not specify a definition of efficiency but discuss it for each scheme individually.

\section{Malicious Server or Semi-honest Client Corruptions}

In this section and the following section, we will demonstrate constructions that achieve security against malicious adversaries, as long as there is no simultaneous server-client corruption.

Roadmap. As described in Section 1.2, our plan of action is: 1) define and obtain an mABE scheme; 2) use Goldwasser et al's compiler techniques [26] to achieve attribute-hiding mABE; and 3) show that attribute-hiding mABE implies private MVC.

All of the above primitives are proven secure under a malicious server or semihonestly corrupted clients in this section. Then, in the following Section 4 we show a generic compiler based on non-interactive zero-knowledge proofs, such that any protocol secure against semi-honest corruption of an arbitrary subset of clients, and additionally offering clients perfect privacy from one another, can be transformed into a protocol that is secure against either a malicious server or an arbitrary subset of malicious clients.

For convenience, in the remainder of the section, we focus on the case when only the first client $P_{1}$ learns output, and the remaining clients learn nothing. Based on this, we can obtain a protocol where every party learns outputs through simple parallel repetition. 


\subsection{Multi-sender ABE}

We define a multi-sender, two-outcome ABE scheme. Intuitively, the mABE functionality implements the following: consider $n$ senders and a server. The first sender $\mathrm{P}_{1}$ chooses two messages $m_{0}$ and $m_{1}$, and each $\mathrm{P}_{i}$ for $i \in[n]$ has an attribute $x_{i}$. The goal is for the server to $m_{b}$ where $b=f\left(x_{1}, x_{2}, \ldots, x_{n}\right)$ while keeping $m_{1-b}$ secret. We require the $\mathrm{mABE}$ scheme to be non-interactive, i.e., after an initial preprocessing phase in which the server learns an encoding of the function $f$, in each online phase, each sender sends a single message to the server, and the server can learn $m_{b}$.

We note that our mABE formulation can also be regarded as a generalization of the proxy oblivious transfer (POT) primitive proposed by Choi et al. [15]. We present the definition of POT in Appendix $\mathrm{A}$. In other words, sender $\mathrm{P}_{1}$ obliviously transfers one of $m_{0}$ and $m_{1}$ to the server, where which message is transferred is determined by a policy function $f$ over all senders' attributes.

Figure 2 formally describes the $\mathrm{mABE}$ ideal functionality. We define $\mathrm{mABE}$ for the single-key setting, since our verifiable computation application is inherently single-key.

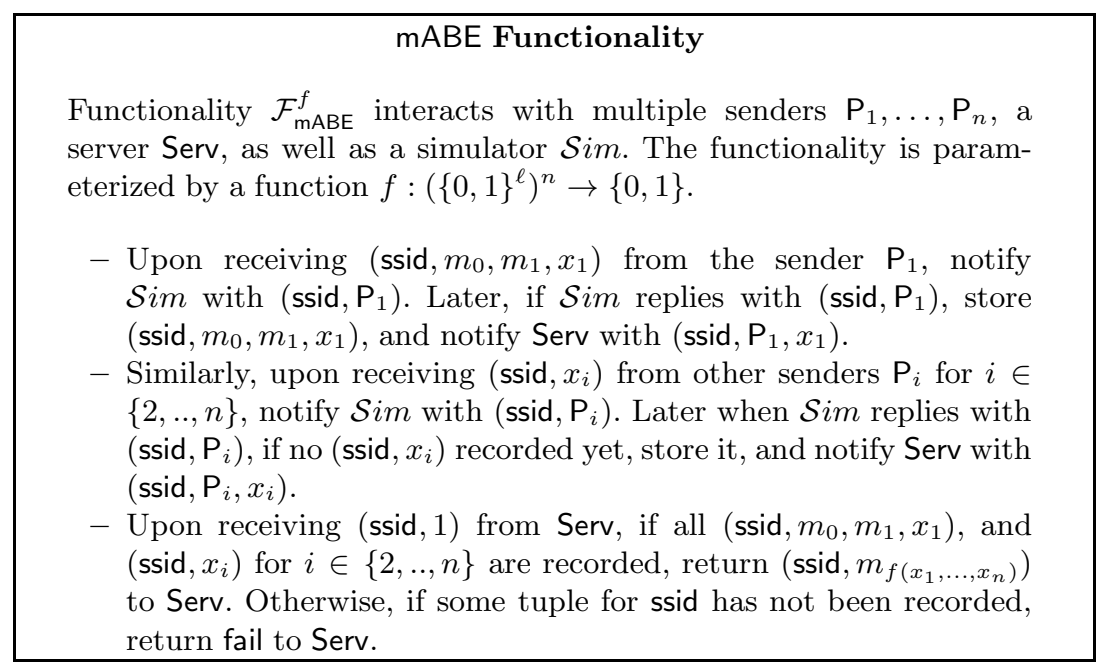

Fig. 2. Functionality $\mathcal{F}_{\text {mABE }}^{f}$

We now present our (non-interactive) protocol that realizes $\mathcal{F}_{\text {mABE }}^{f}$ for any efficiently computable $f$. We use as building blocks a non-interactive POT protocol, and any two-outcome attribute-based encryption (ABE) scheme with a special structure where the attributes of ciphertexts can be encoded bit-by-bit. We formalize this local encoding property in the following. and observe that the ABE construction by Gorbunov, Vaikuntanathan, and Wee [30] satisfies this special property. Also, we remark that one can build a two-outcome ABE from a standard one, as shown by Goldwasser et al. [26]. Here we use ABE to denote the two-outcome ABE for simplicity. 
Definition 3 (Two-outcome ABE with Local Encoding). A two-outcome attribute-based encryption scheme ABE for a class of boolean functions $\mathcal{F}=\left\{\mathcal{F}_{\ell}\right\}_{\ell \in \mathbb{N}}$ from $\{0,1\}^{k} \rightarrow\{0,1\}$, is a tuple of polynomial time algorithms: ABE. $\{$ Setup, KeyGen, Enc, Dec $\}$ as follows:

- ABE.Setup $\left(1^{k}\right)$ outputs a master public key mpk $\mathrm{ABE}$ and a master secret key msk $k_{A B E}$.

- ABE.KeyGen $\left(\operatorname{msk}_{\mathrm{ABE}}, f\right)$ On inputs msk $_{\mathrm{ABE}}$ and a function $f \in \mathcal{F}$, output a function key $\mathrm{sk}_{f}$.

- ABE.Enc $\left(\operatorname{mpk}_{\mathrm{ABE}}, x, m_{0}, m_{1}\right)$ takes as input the master public key $\mathrm{mpk} \mathrm{ABE}_{\mathrm{AB}}$, an attribute $x \in\{0,1\}^{\ell}$ for some $\ell$, and two messages $m_{0}, m_{1}$, outputs a ciphertext $c$.

- ABE.Dec $\left(\mathrm{sk}_{f}, c\right)$ takes as input a key $\mathrm{sk}_{f}$ and a ciphertext and outputs a message $m^{*}$.

Local encoding. We say that a two-outcome ABE scheme satisfies local encoding if the encryption algorithm ABE.Enc can be equivalently expressed as the following, where enc is a sub-algorithm:

1. select common randomness $R$;

2. for all $i \in[k]$, compute $\hat{x}[i]=\operatorname{enc}\left(\operatorname{mpk}_{\mathrm{ABE}}, x[i] ; R\right)$;

3. $\hat{m}=\operatorname{enc}\left(\mathrm{mpk}_{\mathrm{ABE}}, m_{0}, m_{1} ; R\right)$.

Finally, the ciphertext $c$ can be written as $c:=(\hat{x}[1], \hat{x}[2], \ldots, \hat{x}[k], \hat{m})$.

The correctness property guarantees that the decryptor can learn one of the messages $m_{b}$ for $b=f(x)$, and the security guarantees that this is the only thing he can learn. We present the formal definitions in the appendix and also refer the readers to the work by Goldwasser et al. 26.

We present our construction of $\mathrm{mABE}$ in the $\mathcal{G}^{\mathrm{ABE}}$ setup model, where $\mathcal{G}^{\mathrm{ABE}}$ serves as a self-registered PKI which allows the sender to generate $\left(\mathrm{mpk}_{\mathrm{ABE}}\right.$, $\left.\mathrm{msk}_{\mathrm{ABE}}\right) \leftarrow \mathrm{ABE} . \operatorname{KeyGen}\left(1^{k}\right)$, and register $\mathrm{mpk}_{\mathrm{ABE}}$. When queried by players other than the sender, it returns $\mathrm{mpk}_{\mathrm{ABE}}$.

Construction of mABE. Let $f:\left(\{0,1\}^{\ell}\right)^{n} \rightarrow\{0,1\}$ be a policy function, and without loss of generality, we let Serv denote the server, and let $\mathrm{P}_{1}, \ldots, \mathrm{P}_{n}$ denote the senders. We make use of $(n-1) \cdot \ell$ instances of the functionality $\mathcal{F}_{\mathrm{POT}}$ indexed by $(i, j)$ such that for $i \in\{2, \ldots, n\}$, all $j \in[\ell]$, in the $(i, j)$-th instance, $\mathrm{P}_{1}$ plays the sender, $\mathrm{P}_{i}$ plays the chooser, and Serv plays the server. In the protocol below, we assume the existence of private channels; i.e. we assume that all parties encrypt their messages before sending them. This step is left implicit 2 The parties act as follows:

${ }^{2}$ Recall that our protocol for realizing $\mathcal{F}_{\text {РО }}$ relies on a setup phase for establishing a PKI, so we could rely on this PKI for encrypting messages. If we instead were to use a protocol for $\mathcal{F}_{\text {POT }}$ that did not rely on a PKI, we could simply add the establishment of a PKI to the setup phase of this protocol. Finally, we note that the assumption of private channels is not necessary: we could instead choose to leak $\mathrm{P}_{n+1}$ 's output to an eavesdropper. This would suffice for our purposes, but makes the resulting ideal functionality and the security proof a bit more involved. 
- Offline Stage: Every party receives a function $f$ as input. $\mathrm{P}_{1}$ calls the setup $\mathcal{G}^{\mathrm{ABE}}$ to receive $\left(\mathrm{mpk}_{\mathrm{ABE}}, \mathrm{msk}_{\mathrm{ABE}}\right)$, and computes some $\mathrm{sk}_{f}=$ ABE.KeyGen $\left(\operatorname{msk}_{\mathrm{ABE}}, f\right)$. He sends $\mathrm{sk}_{f}$ to the server. All the other clients runs an empty step.

\section{- Online Stage:}

- On input (sid, $m_{0}, m_{1}, x_{1}$ ), the sender $\mathrm{P}_{1}$ does the following in parallel:

1. Sample a random string $R$. Compute $C=\operatorname{enc}\left(\operatorname{mpk}_{\mathrm{ABE}}, m_{0}, m_{1} ; R\right), \hat{x}_{1}=$ enc $\left(\mathrm{mpk}_{\mathrm{ABE}}, x_{1}, R\right)$ (bit-by-bit) and sends them to the receiver Serv.

2. For $i \in\{2, \ldots, n\}, j \in[\ell], \mathrm{P}_{1}$ computes $\hat{c}_{i, j, 0}=\operatorname{enc}\left(\operatorname{mpk}_{\mathrm{ABE}}, 0 ; R\right)$, and $\hat{c}_{i, j, 1}=\operatorname{enc}\left(\operatorname{mpk}_{\mathrm{ABE}}, 1 ; R\right)$, and then sends $\left(\hat{c}_{i, j, 0}, \hat{c}_{i, j, 1}\right)$ to the $(i, j)$-th instance of $\mathcal{F}_{\text {POT }}$.

- For $i \in\{2, \ldots, n\}$, upon receiving (sid, $x_{i}$ ), the party $\mathrm{P}_{i}$ sends, in parallel, $x_{i}[j]$ to the $(i, j)$-th instance of $\mathcal{F}_{\mathrm{POT}}$ for all $j \in[\ell]$. Here $x_{i}[j]$ denotes the $j$-th bit of $x_{i}$.

- Party Serv receives enc $\left(\mathrm{mpk}_{\mathrm{ABE}}, m_{0}, m_{1}\right)$, enc $\left(\mathrm{mpk}_{\mathrm{ABE}}, x_{1}\right)$ (bit-by-bit) from the sender $\mathrm{P}_{1}$, and enc $\left(\mathrm{mpk}_{\mathrm{ABE}}, x_{2}\right), \ldots$, enc $\left(\mathrm{mpk}_{\mathrm{ABE}}, x_{n}\right)$ (bit-by-bit) via the instances of the functionality $\mathcal{F}_{\text {POT }}$. He outputs $m^{\prime}$ by running the ABE decryption algorithm on the received ciphertexts using decryption key $\mathrm{sk}_{f}$.

Then we are able to achieve the following theorem. We present the proof in the full version of this paper.

Theorem 1. Assuming the existence of two-outcome ABE for a function $f:\left(\{0,1\}^{\ell}\right)^{n} \rightarrow\{0,1\}$ with the additional encoding property as above, then the protocol above securely realizes the ideal functionality $\mathcal{F}_{\mathrm{mABE}}^{f}$ in the $\left(\mathcal{F}_{\mathrm{POT}}, \mathcal{G}^{\mathrm{ABE}}\right)-$ hybrid model, against either (1) malicious server corruption, or (2) any semi-honest (static) corruption among any fixed set of clients.

Using $\mathrm{mABE}$ as a building block, we can easily achieve verifiable computation without privacy. In the full version of this paper, we present the formal definition of MVC without privacy, and the protocol that achieves this notion using mABE. We note that the construction is very similar to the one in the next section (see Theorem 3).

\subsection{Achieving Attribute Hiding}

In Figure 3, we define an attribute-hiding version of mABE, where the sender attributes are not leaked to the receiver. The attribute-hiding $m A B E$ functionality, denoted $\mathcal{F}_{\text {ah-mABE}}$, is defined in almost the same way as $\mathcal{F}_{\text {mABE}}$, except that when the functionality notifies the server, it only notifies (ssid, $P_{i}$ ), without leaking the attributes $x_{i}$ 's.

We present our protocol that realizes $\mathcal{F}_{\text {ah-mABE }}$ in the $\mathcal{G}^{\mathrm{FHE}}$ setup plus $\mathcal{F}_{\text {mABE }}$ hybrid model, where $\mathcal{G}^{\mathrm{FHE}}$ serves as a self-registered PKI which allows the sender to generate $\left(\mathrm{pk}_{\mathrm{FHE}}, \mathrm{sk}_{\mathrm{FHE}}\right) \leftarrow \mathrm{FHE} . \operatorname{KeyGen}\left(1^{k}\right)$, and register $\mathrm{pk}_{\mathrm{FHE}}$. When queried by parties other than the sender, it returns $\mathrm{pk}_{\mathrm{FHE}}$. Our construction can be viewed as a distributed version of that of Goldwasser et al. 26], who constructed attributehiding $\mathrm{ABE}$ (or functional encryption) from a non-hiding one. Briefly speaking, 


\section{ah-mABE Functionality}

Functionality $\mathcal{F}_{\text {ah-mABE }}^{f}$ interacts with multiple senders $\mathrm{P}_{1}, \ldots, \mathrm{P}_{n}$, a server Serv, as well as a simulator Sim. The functionality is parameterized by a function $f:\left(\{0,1\}^{\ell}\right)^{n} \rightarrow\{0,1\}$.

- Upon receiving (ssid, $m_{0}, m_{1}, x_{1}$ ) from the sender $\mathrm{P}_{1}$, notify Sim with (ssid, $\mathrm{P}_{1}$ ). Later, if $\mathcal{S}$ im replies with (ssid, $\mathrm{P}_{1}$ ), store (ssid, $m_{0}, m_{1}, x_{1}$ ), and notify Serv with (ssid, $\mathrm{P}_{1}$ ).

- Similarly, upon receiving (ssid, $x_{i}$ ) from other senders $\mathrm{P}_{i}$ for $i \in$ $\{2, . ., n\}$, notify $\mathcal{S} i m$ with $\left(\right.$ ssid, $\left.\mathrm{P}_{i}\right)$. Later when $\mathcal{S}$ im replies with (ssid, $\mathrm{P}_{i}$ ), if no (ssid, $x_{i}$ ) recorded yet, store it, and notify Serv with (ssid, $\mathrm{P}_{i}$ ).

- Upon receiving (ssid, 1) from Serv, if all (ssid, $m_{0}, m_{1}, x_{1}$ ), and (ssid, $\left.x_{i}\right)$ for $i \in\{2, . ., n\}$ are recorded, return (ssid, $m_{f\left(x_{1}, \ldots, x_{n}\right)}$ ) to Serv. Otherwise, if some tuple for ssid has not been recorded, return fail to Serv.

Fig. 3. Functionality $\mathcal{F}_{\text {ah-mABE }}^{f}$

the first party $P_{1}$ generates a garbled circuit of the FHE decryption circuit, and then all parties input ciphertexts of their attributes to $\mathcal{F}_{\mathrm{mABE}}$, to allow the server to learn only a set of labels to the garbled circuit. Then the server can learn only the outcome by evaluating the garbled circuit. Intuitively, since the attributes are encrypted, and the server can learn only a set of labels of the garbled circuit, the server can only learn the outcome but not the attributes of the parties.

Construction of ah-mABE. Let $f:\left(\{0,1\}^{\ell}\right)^{n} \rightarrow\{0,1\}$ be a policy function, let $\mathrm{P}_{1}, \ldots, \mathrm{P}_{n}$ be the senders, and let Serv be the receiver. Denote $g:=$ Eval $_{\mathrm{FHE}}\left(\mathrm{pk}_{\mathrm{FHE}}, f^{\prime},\left(c, c^{\prime}, c_{1}\right), \ldots, c_{n}\right)$ where $\mathrm{pk}_{\mathrm{FHE}}$ is an FHE public key, $c, c^{\prime}, c_{1} \ldots, c_{n}$ are ciphertexts and $f^{\prime}$ is an $n$-nary function that on input $\left(\left(m_{0}, m_{1}, x_{1}\right), \ldots, x_{n}\right)$ outputs $m_{f\left(x_{1}, \ldots, x_{n}\right)}$. Assume the function $g$ has an $\lambda$-bit output, and denote $g_{i}$ as the function that outputs the $i$-bit of $g$. Then the parties do as follows:

- Upon receiving input (ssid, $\left.m_{0}, m_{1}, x_{1}\right), \mathrm{P}_{1}$ does the following:

- Obtain ( $\left.\mathrm{pk}_{\mathrm{FHE}}, \mathrm{sk}_{\mathrm{FHE}}\right)$, and compute $\left(\Gamma,\left\{L_{i}^{0}, L_{i}^{1}\right\}_{i \in[\lambda]}\right) \leftarrow$ Gb.Garble $\left(1^{k}, \operatorname{Dec}_{\mathrm{FHE}}\left(\operatorname{sk}_{\mathrm{FHE}}, \cdot\right)\right)$ where $\operatorname{Dec}_{\mathrm{FHE}}\left(\mathrm{sk}_{\mathrm{FHE}}, \cdot\right)$ is a circuit that takes a $\lambda$-bit ciphertext as input and outputs a single bit message.

- Send (ssid, $\Gamma$ ) to the receiver Serv, and in parallel,

- Compute $\hat{m}_{0} \leftarrow \operatorname{Enc}_{\mathrm{FHE}}\left(\mathrm{pk}_{\mathrm{FHE}}, m_{0}\right), \hat{m}_{1} \leftarrow \operatorname{Enc}_{\mathrm{FHE}}\left(\mathrm{pk}_{\mathrm{FHE}}, m_{1}\right), \hat{x}_{1} \leftarrow$ $\operatorname{Enc}_{\mathrm{FHE}}\left(\mathrm{pk}_{\mathrm{FHE}}, x_{1}\right)$, and send (ssid, $\left.L_{j}^{0}, L_{j}^{1},\left(\hat{m}_{0}, \hat{m}_{1}, \hat{x}_{1}\right)\right)$ to the functionality $\mathcal{F}_{\mathrm{mABE}}^{g_{j}}$ for all $j \in[\lambda]$.

- For $i \in[n] \backslash\{1\}$, upon receiving input (ssid, $x_{i}$ ), $\mathrm{P}_{i}$ first calls $\mathcal{G}^{\mathrm{FHE}}$ to obtain $\mathrm{pk}_{\mathrm{FHE}}$. Then he computes $\hat{x}_{i} \leftarrow \operatorname{Enc}_{\mathrm{FHE}}\left(\mathrm{pk}_{\mathrm{FHE}}, x_{i}\right)$ and sends (ssid, $\left.\hat{x}_{i}\right)$ to the functionality $\mathcal{F}_{\mathrm{mABE}}^{g_{j}}$ for all $j \in[\lambda]$. 
- Upon receiving input (ssid, $\left.\hat{x}_{1}, \ldots, \hat{x}_{n},\left\{L^{d_{i}}\right\}_{i \in[\lambda]}, \Gamma\right)$ from the ideal functionalities and $\mathrm{P}_{1}$, the receiver Serv computes $\operatorname{Gb} . \operatorname{Eval}\left(\Gamma,\left\{L^{d_{i}}\right\}_{i \in[\lambda]}\right)$, and outputs the result of the evaluation.

Then we are able to achieve the following theorem. We present the proof in the full version of this paper.

Theorem 2. Assuming the existence of a fully homomorphic encryption scheme and a garbling scheme, the protocol above securely realizes the ideal functionality $\mathcal{F}_{\text {ah-mABE }}^{f}$ for any efficiently computable $f$ in the $\left(\mathcal{F}_{\mathrm{mABE}}, \mathcal{G}^{\mathrm{FHE}}\right)$-hybrid model, against either (1) malicious server corruption, or (2) semi-honest (static) corruption among any fixed set of senders.

Using the functionality $\mathcal{F}_{\text {ah-mABE}}$, we are able to build an MVC scheme that also achieves input and output privacy, in a similar fashion that (single-sender) privateindex functional encryption implies private verifiable computation [26]. As before, we assume $f$ outputs only one bit and only the first party receives the output. The construction is in the $\mathcal{F}_{\text {ah-mABE }}^{f}$ hybrid model. More formally, let $f:\left(\{0,1\}^{\ell}\right)^{n} \rightarrow$ $\{0,1\}$ be a function to be delegated, let $P_{1}, \ldots, P_{n}$ be the clients and Serv be the server. The the parties do as the following:

- Upon receiving input (ssid, $x_{1}$ ), $\mathrm{P}_{1}$ samples two random inputs $m_{0}, m_{1} \leftarrow$ $\{0,1\}^{\ell}$ and sends (ssid, $\left.m_{0}, m_{1}, x_{1}\right)$ to the functionality $\mathcal{F}_{\text {ah-mABE }}^{f}$. Locally, he stores $m_{0}, m_{1}$.

- For $i \in[n] \backslash\{1\}$, upon receiving message (ssid, $x_{i}$ ), $\mathrm{P}_{i}$ sends (ssid, $x_{i}$ ) to the functionality $\mathcal{F}_{\text {ah-mABE}}^{f}$.

- Upon receiving (ssid, $m$ ) from $\mathcal{F}_{\text {ah-mABE }}^{f}$, the server sends $\mathrm{P}_{1}$ the message (ssid, $m$ ).

- Upon receiving (ssid, $m$ ) from the server, $\mathrm{P}_{1}$ checks whether $m=m_{b}$ for some $b \in\{0,1\}$. If so, he outputs $b$, and otherwise he outputs $\perp$.

In particular we show the following theorem. We present the proof in the full version of this paper.

Theorem 3. The protocol above securely realizes $\mathcal{F}_{\mathrm{pVC}}$ in the $\mathcal{F}_{\text {ah-mABE }}^{f}$ hybrid world, against either (1) malicious server corruption, or (2) semi-honest (static) corruption of any fixed set of clients.

Remark 2. Actually the above theorem can be more general - we can show that the protocol is secure against any (static) pattern of corruption in the $\mathcal{F}_{\text {ah-mABE }}^{f}$ hybrid world (we will include this in the proof). However, in the previous Theorems 1 and 2, we only know how to realize $\mathcal{F}_{\text {ah-mABE }}^{f}$ against either (1) malicious server corruption, or (2) semi-honest (static) corruption of any fixed set of clients. Therefore, by putting things together we can obtain an input-private verifiable computation ( $\mathrm{pVC}$ ) protocol against such patterns of corruption. In Section 5 , we will show that the corruption pattern cannot be extended - it is impossible to construct general pVC protocols against arbitrary server-client collusions. This in particular implies that it is impossible to construct a protocol for $\mathcal{F}_{\text {ah-mABE }}^{f}$ against arbitrary server-client collusions. 
Efficiency of our construction. We outline the efficiency of a scheme where every client receives 1 bit of output — this can be achieved by a parallel repetition of our basic construction where only $\mathrm{P}_{1}$ receives output. For such a private MVC scheme, the server runs in poly $(\kappa) \cdot O(|f| \cdot n)$. If we instantiate using the ABE construction of Gorbunov et al. [30], the run-time and the communication cost for each client is $O(d \cdot n \ell \kappa)$, where $d$ is the depth of the function $f$ being delegated, $\ell$ is the input length, and $\kappa$ is the security parameter. In Appendix B we also offer more detailed discussion. We note that if some non-falsifiable assumption is used, it is possible to remove the dependence on the circuit depth. As mentioned, the focus of this paper is on using falsifiable assumptions.

Also we note that efficiency of Choi et al.'s construction [15] does not depend on circuit depth — however they security is weaker in many respects. An interesting direction for future research is to construct a scheme (or prove impossibility) where the client online computation and communication does not depend on the number of parties $n$ and the circuit depth $d$, by only using standard assumptions.

\section{From Semi-honest to Malicious Clients Corruptions}

In the previous section, we considered the case where the clients can be corrupted in the semi-honest way. In this section, we present a simple compiler that upgrades the previous protocol to one that is secure against any maliciously corrupted clients, and remains non-interactive. That is, the resulting protocol is secure against either malicious server, or against a set of malicious clients. Our construction only needs an additional setup $\mathcal{F}_{\text {CRS }}$.

We note that if we allow more rounds of communication, it is already known how to achieve security against arbitrary malicious corruptions (i.e. of clients and/or the server) 2]. However in the non-interactive multi-client verifiable computation MVC, there are no known constructions. We have already demonstrated security against a malicious server, and we will consider arbitrary corruptions of both the server and the clients in Section 5. Here we address the case where multiple clients are corrupted, and demonstrate that if an MVC protocol offers security against the semi-honest corruption of an arbitrary subset of the clients, and, additionally it offers the clients perfect privacy from one another (as defined in Definition 4), then there exists a simple compiler for guaranteeing security against the malicious corruption of clients. Of course, if we are allowed for a trusted PKI during the setup phase, we could include honestly generated, committed randomness for each party, and then use a NIZK to prove that all messages were honestly generated. However, we are interested in avoiding the use of trusted PKI, instead allowing each party to register the key of their choice; see Remark1 for a discussion about trusted PKI and self-registered PKI.

Definition 4. An MVC protocol $\Pi$ has perfect client privacy if for all inputs $x_{1}, \ldots, x_{n}$, for an adversary $\mathcal{A}$ that semi-honestly corrupts some subset of the parties $\left\{\mathrm{P}_{i}\right\}_{i \in \mathcal{I}}$ where $\mathcal{I} \subset[n]$, and for every random tape $r_{\mathcal{A}}$ belonging to $\mathcal{A}$, there exists a simulator $\mathcal{S}$ im such that the following distributions are identical

$$
\left\{\operatorname{View}_{\Pi\left(x_{1}, \ldots, x_{n}\right), \mathcal{A}}\right\} \equiv\left\{\mathcal{S i m}\left(\left\{x_{i}, y_{i}\right\}_{i \in \mathcal{I}}, r_{\mathcal{A}}\right)\right\}
$$


where $\left(y_{1}, \ldots, y_{n}\right) \leftarrow f\left(x_{1}, \ldots, x_{n}\right)$, and $\mathrm{View}_{\Pi\left(x_{1}, \ldots, x_{n}\right), \mathcal{A}}$ is the view of the adversary when the inputs to the clients are $\left(x_{1}, \ldots, x_{n}\right)$. In particular, the view contains random string $r_{\mathcal{A}}$, inputs $\left\{x_{i}\right\}_{i \in \mathcal{I}}$, and the message received from the server, and the messages generated by honest clients.

Note that what distinguishes this from a standard requirement for semi-honest corruption is that we require indistinguishability to hold for every random tape of the adversary, rather than only on average. Intuitively, if a protocol meets this requirement, we can simplify the standard compilation techniques, since the adversary is free to use the random tape of his choice. To achieve security in the presence of malicious adversaries, it suffices to have the clients prove (using a NIZK) that their messages are consistent with some random string. Formally, we are able to achieve the following theorem. We present the proof in the full version of this paper.

Theorem 4. Suppose there exists an MVC protocol $\Pi$ in self-registered PKI setup hybrid model that is secure against semi-honest client corruptions, and that $\Pi$ has perfect client privacy. Then there exists an MVC protocol $\Pi^{\prime}$ in the $Z K$ and the selfregistered PKI setup hybrid model, which is secure against malicious client corruptions.

In order to apply the compiler results to our protocol, we need to show that our constructions have the desired property. We show this by the following claim:

Claim. If the underlying ABE and FHE and the garbling schemes is perfectly correct, then the private MVC protocol from Section 3 has perfect client privacy.

Proof. Since $\mathrm{P}_{2}, \ldots, \mathrm{P}_{n}$ do not receive messages or output, their views can be simulated easily. Now, we give a simulation of $\mathrm{P}_{1}$ 's view. In the honest protocol, $\mathrm{P}_{1}$ samples random strings $m_{0}, m_{1}$ and some $r$ for generating ciphertexts of the ABE, FHE and garbling schemes. Suppose these schemes have perfect correctness. Then for every $r$ the honest $\mathrm{P}_{1}$ will receive either $m_{0}$ or $m_{1}$ from the server, depending on $b:=f\left(x_{1}, \ldots, x_{n}\right)$. Therefore, given the result of the computation, $b$, and the random tape of $\mathrm{P}_{1}, R=\left(m_{0}, m_{1}, r\right)$, the simulator can simply output $m_{b}$ as the message from the server, producing an identical view. This completes the simulation of his view.

As a consequence of this theorem and Theorems 1, 2, 3, and the fact that noninteractive ZK can be implemented in the CRS model, we are able to construct a private MVC protocol using CRS and self-registered PKI. We summarize this by the following theorem:

Theorem 5. Assume the existence of a fully homomorphic encryption scheme, a garbling scheme, and an ABE that has local encoding property. Assume the primitives have perfect correctness. Then for any efficiently computable $f$, there exists an MVC protocol that securely realizes the ideal functionality $\mathcal{F}_{\mathrm{pVC}}^{f}$ in the CRS and self-registered PKI hybrid model, against (1) any malicious server corruption, or (2) any malicious (static) corruption among any fixed set of clients. 


\section{When the Server and Some Clients Are Corrupted}

In this section, we consider the remaining, more complicated case where the server and clients can be corrupted at the same time. We show that even for a seemingly simple case where only one client and the server are corrupted together, it is impossible to construct private MVC for general functions, under a large class of instantiations of $\mathcal{G}$ setup including trusted PKI (which is stronger than self-registered PKI, see Remark 1), CRS, shared secret randomness, etc. The lower bound holds even in the standalone setting, and for semi-honest corruptions.

In particular, we consider the case with two clients and one server, where the function being delegated is a universal circuit $U(\cdot, \cdot)$, the first client's input is a circuit $C$, the second client's is a string $x$. The server returns $U(C, x)=C(x)$ to both parties. If there exists a private MVC protocol with respect to such $U$, i.e. if there exists a protocol that realizes $\mathcal{F}_{\mathrm{pVc}}$, then, even if it is only secure against semi-honest corruption and only in the standalone setting, we can construct an obfuscator for any circuit. (We refer the reader to the remark following Definition 2 for a definition of the standalone setting.) By previous lower bounds for obfuscation [4], this leads to an impossibility result. We present the formal statement below.

We note that there is a similar lower bound argument in the server-aided MPC setting in the work [2]. Our lower bound further shows that even a natural relaxation of security (where the ideal functionality can be called multiple times if there is server-client corruption) is not achievable for all functionalities.

Theorem 6. Suppose there exist an instantiation of $\mathcal{G}$ setup and a private MVC protocol $\Pi$ (i.e., one that realizes $\mathcal{F}_{\mathrm{pVC}}$ ) for all efficiently computable functions in the $\mathcal{G}$ setup hybrid world, against semi-honest corruptions for arbitrary parties in the standalone model, then there exists an obfuscator for any circuit $C$ secure under the virtual black-box simulation.

Proof. Consider the case where two clients want to delegate the computation of the universal circuit $U(\cdot, \cdot)$ to the server; the first client provides a circuit $C$, and the second provides an input $x$. Then the honest server returns $C(x)$ to both parties. Suppose there exists an instantiation of setup $\mathcal{G}$ and a secure protocol $\Pi$ that achieve this goal, then we construct an obfuscator $O$ that on input $C$ does the following:

- $O$ simulates the setup $\mathcal{G}$ and the role of each client in the offline stage to obtain pub, $\mathrm{sk}_{1}, \mathrm{sk}_{2}, \hat{f}$.

- $O$ simulates the first client's procedure on input $C$ in the online stage. Let $\hat{C}$ be the message that $\mathrm{P}_{1}$ sends to the server.

- $O$ outputs $(\hat{C}$, pub, sk $2, \hat{f})$ as an obfuscation of $C$, i.e. $O(C)$.

To evaluate $O(C)$ on input $x$, the evaluator simulates $\mathrm{P}_{2}$ 's online phase to create an encoding of $x$ using pub, sk $\mathrm{s}_{2}$, and then simulates the (corrupted) server to evaluate $\hat{C}, \hat{x}$ with the encoded version of the function $\hat{f}$.

The correctness follows immediately from the correctness of the protocol $\Pi$, and the efficiency of the obfuscator follows directly from the efficiency of the parties in the protocol $\Pi$. In the rest of the proof, we are going to show the virtual 
black-box (VBB) simulation property. In particular we will turn the protocol simulator into a VBB obfuscation simulator.

Now we analyze the construction. In particular, we want to show given an adversary $\mathcal{A}$ attacking the security of the obfuscation, we are going to construct a simulator $\mathcal{S}$ im such that the probability $\mathcal{A}(O(C))=1$ is close to that of $\mathcal{S i m}^{C}\left(1^{k}\right)=1$ up to a negligible factor for all polynomial-sized circuits $C$. We do this by defining a particular adversary $\mathcal{A}^{*}$ that attacks $\Pi^{\mathcal{G}}$, and using the protocol simulator that is guaranteed to exist for this adversary by the security of the MVC protocol.

Given $\mathcal{A}$ and any poly-sized circuit $C$, we define the following experiment in the $\mathcal{G}$-hybrid world. Let $\mathcal{Z}^{*}$ be an environment and $\mathcal{A}^{*}$ be an adversary attacking protocol $\Pi^{\mathcal{G}}$. $\mathcal{A}^{*}$ corrupts the server and the second party at the beginning. He queries the ideal functionality $\mathcal{G}$ and stores the reply (pub, sk $\mathrm{s}_{2}$ ). Upon receiving a message from $P_{1}$ during the offline stage (on behalf of the server), he uses this message, along with the offline message that an honest $P_{2}$ would send, to construct $\hat{f}$ as the server would do. When $\mathrm{P}_{1}$ sends a message $\hat{C}$ to the server during the online phase, $\mathcal{A}^{*}$ interprets $(\hat{C}$, pub, sk,$\hat{f})$ as an obfuscation of $O(C)$. Then $\mathcal{A}^{*}$ runs $\mathcal{A}$ on the interpreted $O(C)$ and passes $\mathcal{A}$ 's output to $\mathcal{Z}^{*} . \mathcal{Z}^{*}$ outputs this as

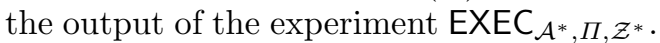

Now we are ready to construct the simulator $\mathcal{S} i m$. By the premise that $\Pi^{\mathcal{G}}$ realizes $\mathcal{F}_{\mathrm{pVC}}$, for this $\mathcal{A}^{*}$, there exists $\mathcal{S} i m^{*}$ such that for this particular $\mathcal{Z}^{*}$, we have $\operatorname{EXEC}_{\mathcal{A}^{*}, \Pi, \mathcal{Z}^{*}}^{\mathcal{G}} \approx \operatorname{EXEC}_{\mathcal{S}_{i m}^{*}, \mathcal{F}_{\mathrm{pvc}}, \mathcal{Z}^{*}}$. Given such $\mathcal{S}_{i m^{*}}$, we define a simulator $\mathcal{S} i m$ for the VBB obfuscation as follows:

- $\mathcal{S}$ im basically simulates the execution of EXEC $\operatorname{Sim}^{*}, \mathcal{F}_{\mathrm{pvc}}, \mathcal{Z}^{*}$.

- Whenever the protocol simulator $\mathcal{S i m}^{*}$ queries the oracle $O_{U,\{2\}}(\cdot)$ in the ideal functionality with some modified $\mathrm{P}_{2}$ 's input $x_{2}^{\prime}, \mathcal{S}$ im simulates it using a blackbox query to $C$ with input $x_{2}^{\prime}$ and returns $C\left(x_{2}^{\prime}\right)$ to $\mathcal{S i m}^{*}$.

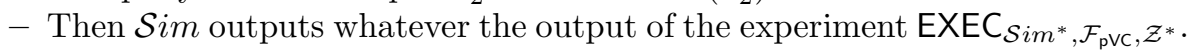

Then we are going to prove that $\mathcal{S} i m$ is a good VBB simulator by the following lemma:

Lemma 1. For the simulator $\mathcal{S}$ im described above, there exists a negligible function $\nu(\cdot)$ such that $\left|\operatorname{Pr}[\mathcal{A}(O(C))=1]-\operatorname{Pr}\left[\operatorname{Sim}^{C}\left(1^{k}\right)=1\right]\right|<\nu(k)$.

Proof. Assume there is a non-negligible function $\varepsilon$ with $\operatorname{Pr}[\mathcal{A}(O(C))=1]-$ $\operatorname{Pr}\left[\operatorname{Sim}^{C}\left(1^{k}\right)=1\right]>\varepsilon$. We show that the real and simulation worlds in the protocol are distinguishable.

According to the description of $\operatorname{EXEC}_{\mathcal{A}^{*}, \Pi, \mathcal{Z}^{*}}^{\mathcal{G}}$, the output of such experiment is identical to that of $\mathcal{A}(O(C))$. On the other hand, the output of $\operatorname{EXEC}_{\mathcal{S i m}^{*}, \mathcal{F}_{\mathrm{pvc}}, \mathcal{Z}^{*}}$ is exactly the same as that of $\mathcal{S i m}^{C}\left(1^{k}\right)$. So this means the executions of the protocol are distinguishable by $\varepsilon$, which reaches a contradiction. Thus we complete the proof. 
Acknowledgments. This research was sponsored in part by the U.S. Army Research Laboratory and the U.K. Ministry of Defence and was accomplished under Agreement Number W911NF-06-3-0001. The views and conclusions contained in this document are those of the authors and should not be interpreted as representing the official policies, either expressed or implied, of the U.S. Army Research Laboratory, the U.S. Government, the U.K. Ministry of Defence, or the U.K. Government. The U.S. and U.K. Governments are authorized to reproduce and distribute reprints for Government purposes notwithstanding any copyright notation hereon.

\section{References}

1. Applebaum, B., Ishai, Y., Kushilevitz, E.: From secrecy to soundness: Efficient verification via secure computation. In: Abramsky, S., Gavoille, C., Kirchner, C., Meyer auf der Heide, F., Spirakis, P.G. (eds.) ICALP 2010. LNCS, vol. 6198, pp. 152-163. Springer, Heidelberg (2010)

2. Asharov, G., Jain, A., López-Alt, A., Tromer, E., Vaikuntanathan, V., Wichs, D.: Multiparty computation with low communication, computation and interaction via threshold FHE. In: Pointcheval, D., Johansson, T. (eds.) EUROCRYPT 2012. LNCS, vol. 7237, pp. 483-501. Springer, Heidelberg (2012)

3. Backes, M., Fiore, D., Reischuk, R.M.: Verifiable delegation of computation on outsourced data. In: Sadeghi, A.-R., Gligor, V.D., Yung, M. (eds.) ACM CCS 2013, pp. 863-874. ACM Press (November 2013)

4. Barak, B., Goldreich, O., Impagliazzo, R., Rudich, S., Sahai, A., Vadhan, S.P., Yang, K.: On the (im)possibility of obfuscating programs. In: Kilian, J. (ed.) CRYPTO 2001. LNCS, vol. 2139, pp. 1-18. Springer, Heidelberg (2001)

5. Bellare, M., Hoang, V.T., Rogaway, P.: Adaptively secure garbling with applications to one-time programs and secure outsourcing. In: Wang, X., Sako, K. (eds.) ASIACRYPT 2012. LNCS, vol. 7658, pp. 134-153. Springer, Heidelberg (2012)

6. Bellare, M., Hoang, V.T., Rogaway, P.: Foundations of garbled circuits. In: Yu, T., Danezis, G., Gligor, V.D. (eds.) ACM CCS 2012, pp. 784-796. ACM Press (October 2012)

7. Ben-Sasson, E., Chiesa, A., Genkin, D., Tromer, E., Virza, M.: SNARKs for C: Verifying program executions succinctly and in zero knowledge. In: Canetti, R., Garay, J.A. (eds.) CRYPTO 2013, Part II. LNCS, vol. 8043, pp. 90-108. Springer, Heidelberg (2013)

8. Ben-Sasson, E., Chiesa, A., Tromer, E., Virza, M.: Succinct non-interactive zero knowledge for a von neumann architecture. In: Usenix Security Symposium (2014)

9. Benabbas, S., Gennaro, R., Vahlis, Y.: Verifiable delegation of computation over large datasets. In: Rogaway, P. (ed.) CRYPTO 2011. LNCS, vol. 6841, pp. 111-131. Springer, Heidelberg (2011)

10. Bitansky, N., Canetti, R., Chiesa, A., Tromer, E.: Recursive composition and bootstrapping for SNARKS and proof-carrying data. In: Boneh, D., Roughgarden, T., Feigenbaum, J. (eds.) 45th ACM STOC, pp. 111-120. ACM Press (June 2013)

11. Brakerski, Z.: Fully homomorphic encryption without modulus switching from classical GapSVP. In: Safavi-Naini, R., Canetti, R. (eds.) CRYPTO 2012. LNCS, vol. 7417, pp. 868-886. Springer, Heidelberg (2012)

12. Canetti, R.: Security and composition of multiparty cryptographic protocols. Journal of Cryptology 13(1), 143-202 (2000) 
13. Canetti, R.: Universally composable security: A new paradigm for cryptographic protocols. Cryptology ePrint Archive, Report 2000/067 (2000), http://eprint.iacr.org/2000/067

14. Canetti, R.: Universally composable security: A new paradigm for cryptographic protocols. In: 42nd FOCS, pp. 136-145. IEEE Computer Society Press (October 2001)

15. Choi, S.G., Katz, J., Kumaresan, R., Cid, C.: Multi-client non-interactive verifiable computation. In: Sahai, A. (ed.) TCC 2013. LNCS, vol. 7785, pp. 499-518. Springer, Heidelberg (2013)

16. Chung, K.-M., Kalai, Y., Vadhan, S.P.: Improved delegation of computation using fully homomorphic encryption. In: Rabin, T. (ed.) CRYPTO 2010. LNCS, vol. 6223, pp. 483-501. Springer, Heidelberg (2010)

17. Chung, K.-M., Kalai, Y.T., Liu, F.-H., Raz, R.: Memory delegation. In: Rogaway, P. (ed.) CRYPTO 2011. LNCS, vol. 6841, pp. 151-168. Springer, Heidelberg (2011)

18. Fiore, D., Gennaro, R.: Publicly verifiable delegation of large polynomials and matrix computations, with applications. In: Yu, T., Danezis, G., Gligor, V.D. (eds.) ACM CCS 2012, pp. 501-512. ACM Press (October 2012)

19. Garg, S., Gentry, C., Sahai, A., Waters, B.: Witness encryption and its applications. In: Boneh, D., Roughgarden, T., Feigenbaum, J. (eds.) 45th ACM STOC, pp. 467476. ACM Press (June 2013)

20. Gennaro, R., Gentry, C., Parno, B.: Non-interactive verifiable computing: Outsourcing computation to untrusted workers. In: Rabin, T. (ed.) CRYPTO 2010. LNCS, vol. 6223, pp. 465-482. Springer, Heidelberg (2010)

21. Gennaro, R., Gentry, C., Parno, B., Raykova, M.: Quadratic span programs and succinct nIZKs without pCPs. In: Johansson, T., Nguyen, P.Q. (eds.) EUROCRYPT 2013. LNCS, vol. 7881, pp. 626-645. Springer, Heidelberg (2013)

22. Gentry, C., Lewko, A.B., Sahai, A., Waters, B.: Indistinguishability obfuscation from the multilinear subgroup elimination assumption. IACR Cryptology ePrint Archive, 2014:309 (2014)

23. Goldreich, O.: Foundations of Cryptography: Basic Applications, vol. 2. Cambridge University Press, Cambridge (2004)

24. Goldwasser, S., Gordon, S.D., Goyal, V., Jain, A., Katz, J., Liu, F.-H., Sahai, A., Shi, E., Zhou, H.-S.: Multi-input functional encryption. In: Nguyen, P.Q., Oswald, E. (eds.) EUROCRYPT 2014. LNCS, vol. 8441, pp. 578-602. Springer, Heidelberg (2014)

25. Goldwasser, S., Kalai, Y.T., Popa, R.A., Vaikuntanathan, V., Zeldovich, N.: How to run turing machines on encrypted data. In: Canetti, R., Garay, J.A. (eds.) CRYPTO 2013, Part II. LNCS, vol. 8043, pp. 536-553. Springer, Heidelberg (2013)

26. Goldwasser, S., Kalai, Y.T., Popa, R.A., Vaikuntanathan, V., Zeldovich, N.: Reusable garbled circuits and succinct functional encryption. In: Boneh, D., Roughgarden, T., Feigenbaum, J. (eds.) 45th ACM STOC, pp. 555-564. ACM Press (June 2013)

27. Goldwasser, S., Lin, H., Rubinstein, A.: Delegation of computation without rejection problem from designated verifier CS-proofs. IACR Cryptology ePrint Archive, 2011:456 (2011)

28. Goldwasser, S., Micali, S.: Probabilistic encryption. J. Comput. Syst. Sci. 28(2), 270-299 (1984)

29. Goldwasser, S., Micali, S., Rackoff, C.: The knowledge complexity of interactive proof systems. SIAM J. Comput. 18(1), 186-208 (1989) 
30. Gorbunov, S., Vaikuntanathan, V., Wee, H.: Attribute-based encryption for circuits. In: Boneh, D., Roughgarden, T., Feigenbaum, J. (eds.) 45th ACM STOC, pp. 545554. ACM Press (June 2013)

31. Kamara, S., Mohassel, P., Raykova, M.: Outsourcing multi-party computation. Cryptology ePrint Archive, Report 2011/272 (2011), http://eprint.iacr.org/

32. Kamara, S., Mohassel, P., Riva, B.: Salus: a system for server-aided secure function evaluation. In: Yu, T., Danezis, G., Gligor, V.D. (eds.) ACM CCS 2012, pp. 797-808. ACM Press (October 2012)

33. Kosba, A.E., Papadopoulos, D., Papamanthou, C., Sayed, M.F., Shi, E., Triandopoulos, N.: Trueset: Nearly practical verifiable set computations. In: Usenix Security Symposium (2014)

34. Papamanthou, C., Shi, E., Tamassia, R.: Signatures of correct computation. In: Sahai, A. (ed.) TCC 2013. LNCS, vol. 7785, pp. 222-242. Springer, Heidelberg (2013)

35. Papamanthou, C., Tamassia, R., Triandopoulos, N.: Optimal verification of operations on dynamic sets. In: Rogaway, P. (ed.) CRYPTO 2011. LNCS, vol. 6841, pp. 91-110. Springer, Heidelberg (2011)

36. Parno, B., Raykova, M., Vaikuntanathan, V.: How to delegate and verify in public: Verifiable computation from attribute-based encryption. In: Cramer, R. (ed.) TCC 2012. LNCS, vol. 7194, pp. 422-439. Springer, Heidelberg (2012)

37. Shi, E., Chan, T.-H.H., Rieffel, E.G., Chow, R., Song, D.: Privacy-preserving aggregation of time-series data. In: NDSS 2011. The Internet Society (February 2011)

\section{A Preliminaries}

Here we present the definitions we use in this paper.

\section{A.1 Two-outcome ABE}

Definition 5 (Correctness of Two-outcome ABE [26]). For any polynomial $n(\cdot)$, for every sufficiently large security parameter $\kappa$, if $n=n(\kappa)$, for all boolean functions $f \in \mathcal{F}_{n}$, attributes $x \in\{0,1\}^{n}$, messages $M_{0}, M_{1} \in \mathcal{M}$, there exists some negligible $\nu(\cdot)$ such that

$$
\operatorname{Pr}\left[\begin{array}{l}
\left(m_{\mathrm{ABE}}, \text { msk }_{\mathrm{ABE}}\right) \leftarrow \operatorname{ABE} . \operatorname{Setup}\left(1^{\kappa}\right) ; \\
\text { sk }_{f} \leftarrow \mathrm{ABE} . \operatorname{KeyGen}\left(\mathrm{msk}_{\mathrm{ABE}}, f\right) ; \\
c \leftarrow \mathrm{ABE} . \operatorname{Enc}\left(\mathrm{mpk}_{\mathrm{ABE}}, x, m_{0}, m_{1}\right) ; \\
m=\mathrm{ABE} . \operatorname{Dec}\left(\mathrm{sk}_{f}, c\right): \\
m=m_{f(x)}
\end{array}\right]=1-\nu(\kappa) .
$$

If $\nu=0$, then the scheme has perfect correctness.

Then we define the security for single-key two-outcome ABE.

Definition 6 (Security of Two-outcome ABE [26]). Let ABE be a twooutcome $A B E$ scheme for the class of boolean functions $\mathcal{F}=\left\{\mathcal{F}_{n}\right\}_{n \in \mathbb{N}}$ and associated message space $\mathcal{M}$ and let $\mathcal{A}=\left(\mathcal{A}_{1}, \mathcal{A}_{2}, \mathcal{A}_{3}\right)$ be a triple of PPT adversaries. Consider the following experiment. 
$-\left(\mathrm{mpk}_{\mathrm{ABE}}, \mathrm{msk}_{\mathrm{ABE}}\right) \leftarrow \mathrm{ABE} \cdot \operatorname{Setup}\left(1^{\kappa}\right)$

$-\left(f, \mathrm{st}_{1}\right) \leftarrow \mathcal{A}_{1}\left(\mathrm{mpk}_{\mathrm{ABE}}\right)$

- sk $_{f} \leftarrow$ ABE.KeyGen $\left(\operatorname{msk}_{\mathrm{ABE}}, f\right)$

$-\left(m, m_{0}, m_{1}, x, \mathrm{st}_{2}\right) \leftarrow \mathcal{A}_{2}\left(\mathrm{st}_{1}, \mathrm{sk}_{f}\right)$

- choose a bit $b$ at random. Then let

$$
c=\left\{\begin{array}{l}
\mathrm{ABE} \cdot \operatorname{Enc}\left(\mathrm{mpk}_{\mathrm{ABE}}, x, m, m_{b}\right), \text { if } f(x)=0, \\
\mathrm{ABE} \cdot \operatorname{Enc}\left(\mathrm{mpk}_{\mathrm{ABE}}, x, m_{b}, m\right), \text { otherwise. }
\end{array}\right.
$$

$-b^{\prime} \leftarrow \mathcal{A}_{3}\left(\mathrm{st}_{2}, c\right)$. If $b=b^{\prime}$, and there exists $n$ such that, for all $f \in \mathcal{F}_{n}$, messages $m . m_{0}, m_{1} \in \mathcal{M},\left|m_{0}\right|=\left|m_{1}\right|, x \in\{0,1\}^{n}$, output 1 . Else output 0 .

We say the scheme is a full-secure single-key two-outcome ABE if for all PPT adversaries $\mathcal{A}$, and for all sufficiently large $\kappa$, the probability that the experiment outputs 1 is bounded by $1 / 2+\nu(k)$ for some negligible function $\nu$.

\section{A.2 Garbling Schemes}

Definition 7 (Garbling Schemes [6]). A garbling scheme for a family of circuits $C=\left\{C_{n}\right\}_{n \in \mathbb{N}}$ with $C_{n}$ a set of boolean circuits taking as input $n$ bits, is a tuple of PPT algorithms $\mathrm{Gb}=\mathrm{Gb} .\{$ Garble, Enc, Eval\} such that

- Gb.Garble $\left(1^{\kappa}, C\right)$ takes as input the security parameter $\kappa$ and a circuit $C \in C_{n}$ for some $n$ and outputs the garbled circuit $\Gamma$ and a secret key sk.

- Gb.Enc(sk, $x)$ takes as input $x$ and outputs an encoding $c$,

- Gb.Eval $(\Gamma, c)$ takes as input a garbled circuit $\Gamma$ and an encoding $c$, and outputs a value $y$ which should be $C(x)$.

The correctness and efficiency properties are straight-forward. Next we consider a special property of the encoding of the Yao's garbled scheme, which we will use in this paper. The secret key has the form sk $=\left\{L_{i}^{0}, L_{i}^{1}\right\}_{i \in[n]}$, and the encoding of an input $x$ of $n$ bits is of the form $c=\left(L^{x_{1}}, L^{x_{2}}, \ldots, L^{x_{n}}\right)$, where $x_{i}$ is the $i$-th bit of $x$.

Then we are going to define the security of garbling schemes.

Definition 8 (Input and Circuit Privacy). A garbling scheme Gb for a family of circuits $\left\{C_{n}\right\}_{n \in \mathbb{N}}$ is input and circuit private if there exists a PPT simulator Sim such that for every adversaries $\mathcal{A}$ and $D$, for all sufficiently large $\kappa$,

$\operatorname{Pr}\left[\begin{array}{c}(x, C, \alpha) \leftarrow A\left(1^{k}\right) ; \\ (I, \text { sk }) \leftarrow \mathrm{Gb} . G \mathrm{Garble}\left(1^{\kappa}, C\right) ; \\ c \leftarrow \mathrm{Gb} . \text { Enc }(\mathrm{sk}, x): \\ D(\alpha, x, C, \Gamma, c)=1\end{array}\right]-\operatorname{Pr}\left[\begin{array}{c}(x, C, \alpha) \leftarrow A\left(1^{k}\right) ; \\ \left.(\tilde{\Gamma}, \tilde{c}) \leftarrow \mathcal{S i m}\left(1^{\kappa}, C(x), 1^{|C|}, 1^{|x|}\right):\right] \\ D(\alpha, x, C, \tilde{\Gamma}, \tilde{c})=1\end{array}\right] \mid=\nu(k)$

for some negligible $\nu(\cdot)$, where we consider only $\mathcal{A}$ such that for somen, $x \in\{0,1\}^{n}$ and $C \in C_{n}$. 


\section{A.3 Extractable Witness Encryption}

Definition 9 (Witness Encryption [19]). A witness encryption for a language $L \in N P$ with corresponding witness relation $R_{L}$ consists of two polynomial-time algorithms WE. \{Enc, Dec $\}$ such that

- Encryption WE.Enc $\left(1^{\kappa}, x, b\right)$ : takes as input a security parameter $\kappa$, a statement $x \in\{0,1\}^{*}$, a bit $b$ and outputs a ciphertext $c$.

- Decryption WE.Dec $(w, c)$ : takes as input a witness $w \in\{0,1\}^{*}$ and a ciphertext $c$ and outputs a bit $b$ or $\perp$.

Correctness: For all $(x, w) \in R_{L}$, for all bits b for every sufficiently large security parameter $\kappa$, we have

$$
\operatorname{Pr}\left[c \leftarrow \operatorname{WE} . \operatorname{Enc}\left(1^{\kappa}, x, b\right): \operatorname{WE} . \operatorname{Dec}(w, c)=b\right]=1-\nu(\kappa)
$$

for some negligible $\nu$.

Definition 10 (Extractable Security [25]). A witness encryption scheme for a language $L \in N P$ is secure if for all PPT adversaries $\mathcal{A}$, and all poly $q$, there exists a PPT extractor $E$ and a poly $p$ such that for all auxiliary input $z$ and all $x \in\{0,1\}^{*}$, the following holds:

$$
\begin{gathered}
\operatorname{Pr}\left[b \leftarrow\{0,1\} ; c \leftarrow \text { WE.Enc }\left(1^{\kappa}, x, b\right): A(x, c, b)=b\right] \geq 1 / 2+1 / q(|x|) \\
\Rightarrow \operatorname{Pr}\left[E(x, z)=w:(x, w) \in R_{L}\right] \geq 1 / p(|x|) .
\end{gathered}
$$

\section{A.4 Obfuscations}

Definition 11 (Circuit Obfuscator [4]). A probabilistic algorithm $O$ is a (circuit) obfuscator for the collection $\mathcal{F}$ of circuits if the following holds:

- (functionality) For every circuit $C \in \mathcal{F}$, the string $O(C)$ describes a circuit that computes the same function as $C$.

- (polynomial slowdown) There is a polynomial $p$ such that for every circuit $C \in$ $\mathcal{F}$, we have $|O(C)| \leq p(|C|)$.

- ("virtual black box" (VBB) property) For any PPT $\mathcal{A}$, there is a PPT Sim and a negligible function $\nu$ such that for all circuits $C \in \mathcal{F}$, it holds that

$$
\left|\operatorname{Pr}[A(O(C))=1]-\operatorname{Pr}\left[\operatorname{Sim}^{C}\left(1^{|C|}\right)=1\right]\right| \leq \nu(|C|) .
$$

We say that $O$ is efficient if it runs in polynomial time. If we omit specifying the collection $\mathcal{F}$, then it is assumed to be the collection of all circuits. 


\section{A.5 Proxy Oblivious Transfer}

Choi et al. [15] recently defined and constructed proxy oblivious transfer. Instead of taking the game based security definitions from the paper by Choi et al., here we define the security of POT in the real/ideal paradigm, which provides a stronger security guarantee. In the ideal functionality below, we omit the session id for notational simplicity. We remark that in each session, the functionality could accept multiple new inputs; we assign a sub-session id, i.e., ssid, for each new input.

Theorem 7 ([15]). There is a non-interactive protocol which realizes $\mathcal{F}_{\mathrm{POT}}$ in

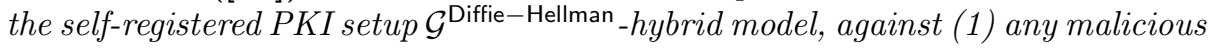
server corruption, or (2) any semi-honest (static) corruption among any fixed set of clients.

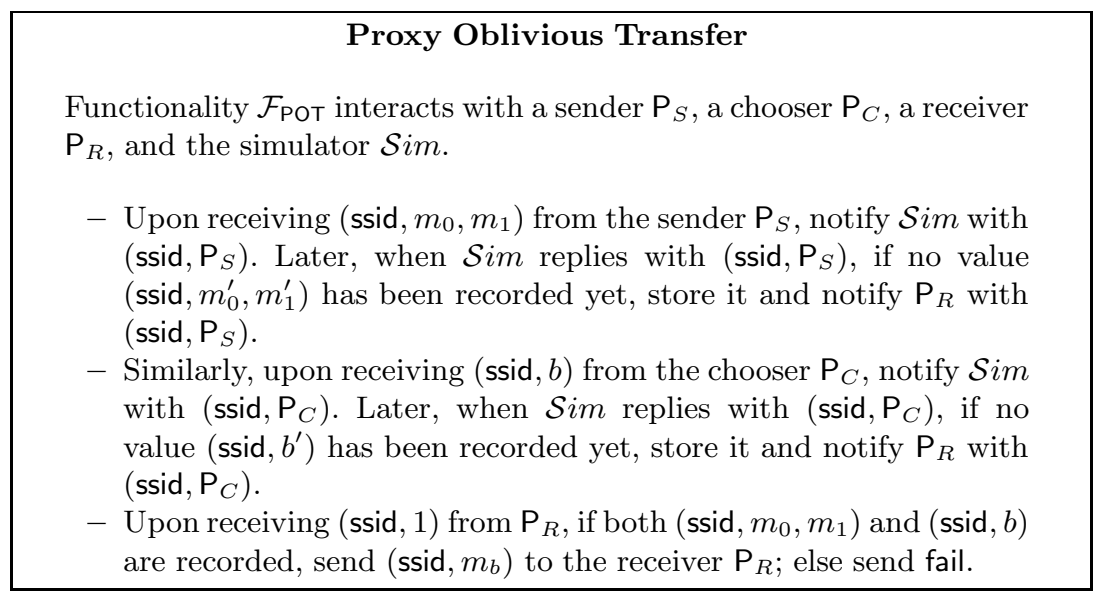

Fig. 4. Functionality $\mathcal{F}_{\text {POT }}$

Choi et al. 15] constructed a non-interactive protocol in the offline/online model that realizes the ideal functionality $\mathcal{F}_{\text {РОт }}$. In this model, the two clients run some protocol in the offline stage, prior to learning their inputs, and then complete the protocol in the online stage, after receiving their inputs. In their construction, the clients do not need to interact in the offline stage, and in the online stage both the sender and chooser send a single message to the server. The construction relies on the existence of non-interactive key agreement schemes (e.g., the Diffie-Hellman key exchange scheme).

\section{B Instantiations and Efficiency}

In this section, we discuss the instantiations of our building blocks. We need a two-outcome attribute encryption scheme with the local encoding property, a fully 
homomorphic encryption scheme, and a garbling scheme. In particular we can use any instantiation of FHE schemes, e.g. one by Brakerski [1], and any instantiation of Yao's garbling scheme.

The attribute based encryption (ABE) constructed by Gorbunov, Vaikuntanathan, Wee [30] actually achieves the requirements of regular ABE with the local encoding property. Goldwasser et al. [26] showed a generic way to achieve two-outcome ABE from a regular one. So by plugging the GSW ABE scheme and using the generic technique, we achieve the two-outcome $\mathrm{ABE}$ as required by Definition 3 ,

For our private MVC scheme, the server clearly runs in poly $(\kappa, f)$. For the clients, $\mathrm{P}_{2}, \ldots, \mathrm{P}_{n}$ runs in time $O(\ell \kappa)$, where $\ell$ is the input length; $\mathrm{P}_{1}$ generates $O(n \ell \kappa)$ ABE ciphertexts plus a garble circuit of size $O(\kappa)$, where $n$ is the number of parties, $\ell$ is the input length, and $\kappa$ is the security parameter. However, the ciphertexts' length for the currently best known ABE construction of Gorbunov, Vaikuntanathan, Wee [30] depends on the circuit depth (independent of the size). Therefore, $\mathrm{P}_{1}$ 's running time (the communication complexity as well) depends on $O(d \cdot n \ell \kappa)$, where $d$ is the depth of the function being delegated. The construction of Choi et al. 15] has better online efficiency for clients that is independent of the function complexity, but has the issues of adaptive soundness and is vulnerable to selective failure attacks. The construction using multi-input functional encryption 24] can achieve better efficiency but their solution inherently requires the existence of indistinguishable obfuscation, which is a stronger assumption and has large overhead. 